\title{
Galactic archeology with extremely metal-poor stars
}

\author{
Yutaka Komiya ${ }^{1}$, Takuma Suda ${ }^{2}$, Asao Habe ${ }^{2}$, \\ and Masayuki Fujimoto ${ }^{2}$ \\ ${ }^{1}$ Department of Astronomy, Faculty of Science, Tohoku University, \\ Sendai, Miyagi Prefecture 980-8578, Japan \\ email: komiya@astro.tohoku.ac.jp \\ ${ }^{2}$ Department of Cosmosciences, Hokkaido University, \\ Sapporo, Hokkaido 060-0810, Japan
}

\begin{abstract}
Extremely metal-poor (EMP) stars are thought to be formed in the low-mass protogalaxies as building blocks of the Milky Way and can be probes to investigate the early stage of galaxy formation and star formation in the early universe. We study the formation history of EMP stars in the Milky Way halo using a new model of chemical evolution based on the hierarchical theory of the galaxy formation. We construct the merging history of the Milky Way halo based on the extended Press-Schechter formalism, and follow the star formation and chemical evolution along the merger tree. The abundance trends and number of low-mass stars predicted in our model are compared with those of observed EMP stars. Additionally, in order to clarify the origin of hyper metal poor stars, we investigate the change of the surface metal abundances of stars by accretion of interstellar matter. We also investigate the pre-enrichment of intergalactic matter by the first supernovae.
\end{abstract}

Keywords. stars: abundances, (stars:) binaries: general, stars: chemically peculiar, stars: formation, stars: luminosity function, stars: mass function

\section{Introduction}

Recent surveys detected thousands of EMP stars in the Milky Way halo (Beers et al. 1992, Christlieb et al. 2001), hundreds of which are observed by high dispersion spectroscopy (e.g. Aoki et al. 2007, Spite et al. 2005). Thanks to these observations, we can discuss the statistical features of EMP stars. These EMP stars are formed in the ancient Galaxy and are useful probes of star formation in the early universe and metal-poor environments. Such a study of the early universe and formation of the Galaxy with metal-poor stars is called Galactic Archaeology. However, we can observe only low-mass survivors among stars formed in the early universe, while massive stars should have ended their lives leaving their nucleosynthetic signatures. Hereafter, we call all the stellar population of $[\mathrm{Fe} / \mathrm{H}] \lesssim-2.5$ "EMP population" and low mass subset "EMP survivors".

One prominent observational feature of EMP survivor is that the fraction of carbonenhanced EMP (CEMP) stars is very large ( $\gtrsim 20 \%$; see e.g., Suda et al. 2008) compared with more metal-rich counterparts (a few \%). In our previous study (Komiya et al. 2007), we showed that CEMP stars are formed as secondary components of binary systems where matter enriched with carbon is transferred from intermediate-mass primary stars. Based on the stellar evolution model of Fujimoto et al. (2000), we can constrain the IMF of EMP stars by requiring the statistical features of CEMP stars to be consistent with model predictions. Observed large fractions of CEMP stars in EMP stars, number ratio between CEMP stars with and without s-process element enhancement, total number of EMP survivors, and metallicity distribution function $(\mathrm{MDF})$ of stars with $-2.5 \gtrsim[\mathrm{Fe} / \mathrm{H}] \gtrsim-4$, 
imply a massive IMF with medium mass $M_{\mathrm{md}} \sim 10 M_{\odot}$ (Komiya et al. 2007, Komiya et al. 2008a). This result suggests that most of EMP survivors are secondary companions of massive or intermediate-mass stars when they were formed.

Two intriguing problems of EMP stars are the scarcity of stars of $[\mathrm{Fe} / \mathrm{H}] \lesssim-4$ and the existence of three hyper metal-poor (HMP) stars, having metallicities $[\mathrm{Fe} / \mathrm{H}]<-4.5$ Christlieb et al. (2002), Frebel et al. (2005), Norris et al. (2007). Karlsson (2005) and Karlsson (2006) insist that this metallicity gap can be interpreted as a result of a metal diffusion process in the stochastic chemical evolution model. On the other hand, some studies advocate a change in the IMF around $[\mathrm{Fe} / \mathrm{H}]=-4$ (Tumlinson 2006, Salvadori et al. 2006). For the origin of HMP stars, Suda et al. (2004) propose the possibility that they are remnant Pop. III stars formed without metals and polluted by the accretion of interstellar matter (ISM) and binary mass transfer. Umeda \& Nomoto (2003) proposed that these stars are formed from the ejecta of the first supernovae with peculiar abundance patterns. Karlsson (2006) point out that the small amount of iron in HMP stars is explained by pre-enrichment by Pop. III stars. In this paper, we investigate the MDF of EMP stars using the model of chemical evolution with a semi-analytic merger tree and explain the origin of the metallicity gap, taking into account hierarchical galaxy formation. We also study two hypotheses for the origin of HMP stars: surface pollution and pre-enrichment by the first supernovae.

\section{Hierarchical Chemical Evolution}

In order to investigate the effects of structure formation processes in the early stages of chemical evolution, we plant a merger tree using the extended Press-Schechter approach (Bond et al. 1991, Lacey \& Cole 1993). We calculate the merger tree of halo with a mass of $10^{12} M_{\odot}$ in a $\Lambda$ CDM universe $\left(\Omega_{M}=1-\Omega_{\Lambda}=0.3, \Omega_{b}=0.045, h=0.7, \sigma_{8}=\right.$ 0.9) with the method constructed in Somerville \& Kolatt (1999). Mass resolution of mini-halos, $M_{h, l}$, is determined by the halo mass when the virial temperature becomes $T_{\text {vir }}\left(M_{h, l}, z\right)=10^{3} \mathrm{~K}$ (Tegmark et al. 1997, Nishi \& Susa 1999). We use the result of Lacey \& Cole (1993) to calculate the time for merged mini-halos orbit as satellites. We use simple assumptions about star formation and chemical evolution. Star formation efficiency is constant: $10^{10} / \mathrm{yr}$ and the binary fraction is $50 \%$. For the EMP population, we assume a log-normal IMF with $M_{\mathrm{md}} \sim 10 M_{\odot}$, derived by Komiya et al. (2007) and flat mass ratio distribution, $n(q)=1$. The stellar yields and lifetimes are taken from Tominaga et al. (2007) and Schaerer (2002), respectively. Metals ejected by the supernovae spread instantaneously and homogeneously in their host halos. For the first stars in the minihalos, we assume that $M_{\mathrm{md}}=50 M_{\odot}$ and the binary fraction is set to 0 , to suppress star formation in the host halo.

Figure 1 shows the resultant MDF. The slope of the MDF as well as the total observed number are consistent with theoretical results in the metallicity range $-2.5 \gtrsim[\mathrm{Fe} / \mathrm{H}] \gtrsim$ -4.5 . The metallicity cut-off at $[\mathrm{Fe} / \mathrm{H}] \sim-4$ is also well reproduced by the hierarchical scenario. In the current model, typical Type II supernovae (SN) eject $\sim 0.07 M_{\odot}$ of iron into the ISM. The metallicity of the primordial halo of typical mass $\left(\sim 10^{6} M_{\odot}\right)$ becomes

$$
[\mathrm{Fe} / \mathrm{H}]=\log \frac{0.07 M_{\odot}}{10^{6} M_{\odot}\left(\Omega_{b} / \Omega_{m}\right)}-\log Z_{\odot} \sim-3.5 .
$$

On average, the next generation of stars is expected to have typical metallicities of $[\mathrm{Fe} / \mathrm{H}] \gtrsim-3.5$ in the mini-halo. If mini-halos have larger mass, stars with metallicities of $[\mathrm{Fe} / \mathrm{H}] \sim-4$ can be formed. Our result suggests that HMP stars with metallicity $[\mathrm{Fe} / \mathrm{H}] \lesssim-4.5$ are likely to be born before the first supernova in the host cloud. 


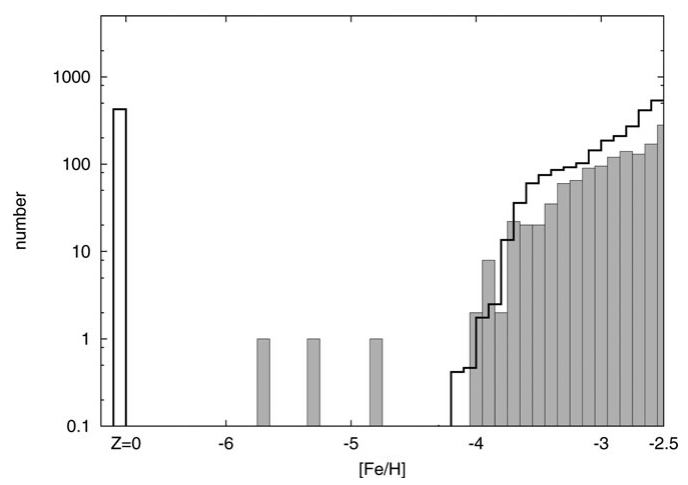

Figure 1. Comparison of the theoretical and observational MDF of EMP stars. The shaded histogram shows the observed number of stars with the HK and HES surveys Beers (2005). The solid line shows the estimated number of stars in the detectable survey areas assuming a uniform distribution of halo stars.

In the lowest metallicity range, however, our numerical results do not agree with the observations. Our model predicts a large number of Pop. III stars. They are second generation stars in the halos with the first stars of mass $m>50 M_{\odot}$. These stars of $M>$ $50 M_{\odot}$ become black holes without iron ejection, and second generation stars without iron are formed. The predicted number of second generation Pop. III stars becomes large because we assume that the IMF of second generation stars with $Z=0$ is the same as EMP stars. In contrast to this result, the observed number of stars with $[\mathrm{Fe} / \mathrm{H}]<-4.5$ is very small. It suggests that the change of IMF from the first stars $\left(M_{\mathrm{md}} \gtrsim 50\right)$ to the EMP population $\left(M_{\mathrm{md}} \sim 10\right)$ is caused by metals, not by radiation from the first stars. But the critical metallicity to enhance the low mass star formation is not necessarily $[\mathrm{Fe} / \mathrm{H}] \sim-4$.

\section{Origin of HMP stars}

\subsection{Accretion of ISM}

EMP stars are likely to modify their surface abundances due to the possible accretion processes during their long lives. In particular, it is pointed out that the small amount of metals in HMP stars comes from interstellar accretion and that they are possibly Pop. III stars polluted by accretion of the ISM (Suda et al. 2004). We trace the modifications of surface abundances in individual low-mass Pop. III or EMP stars which survive to date.

In considering the effect of accretion, we adopt the Bondi-Hoyle accretion rate

$$
\dot{M}=4 \pi(G M)^{2} \rho\left(V_{r}^{2}+c_{s}^{2}\right)^{-3 / 2},
$$

where $M, \rho, c_{s}$, and $V_{r}$ are the stellar mass, the density and the sound velocity of the ISM, and the relative velocity between stars and ambient gas, respectively. We use the following assumptions for dynamics of gas and stars: (1) gas is cooled down to $200 \mathrm{~K}$ and concentrated around the center of the mini-halo; (2) $V_{r}$ is negligible in the mother cloud in which EMP stars are formed; (3) once the mother cloud accretes onto a larger halo, stars move with virial velocity, $V_{\mathrm{v} i r}$, in an ISM of average density $\rho_{\mathrm{av}}=\bar{\rho} \Delta_{c} \Omega_{b}$. (4) All the ISM accreted on binary systems settles onto the EMP survivors, to give the upper limit of the accretion rate.

Figure 2 shows the MDF taking into account surface pollution by accretion of the ISM. Accreted matter is mixed in the surface convective zone of mass of $M_{\mathrm{SCZ}}=0.2 M_{\odot}$ and $0.003 M_{\odot}$ for giant and main sequence stars, respectively (Fujimoto et al. 1995). Surface 


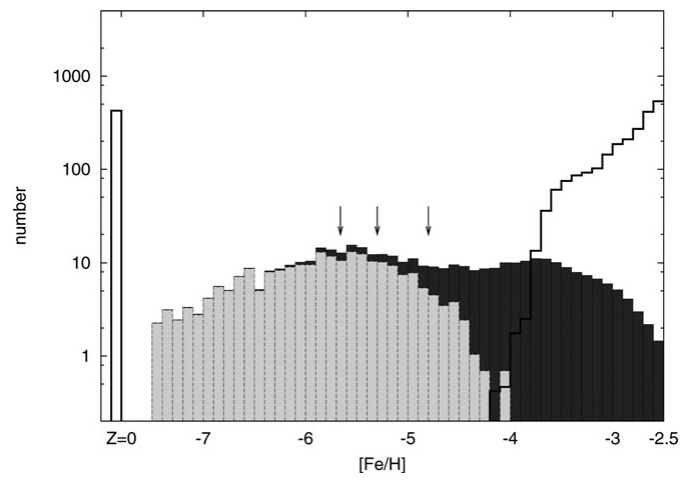

Figure 2. Effect of surface pollution on Pop. III stars and EMP stars. Solid line shows MDF with surface pollution of ISM taken into account. Dark and light gray histograms show the distribution of the polluted main sequence Pop. III stars and giant Pop. III stars, respectively. The different degree of pollution is due to the depth of surface convective zones. Three arrows denote the locations of three HMP stars.
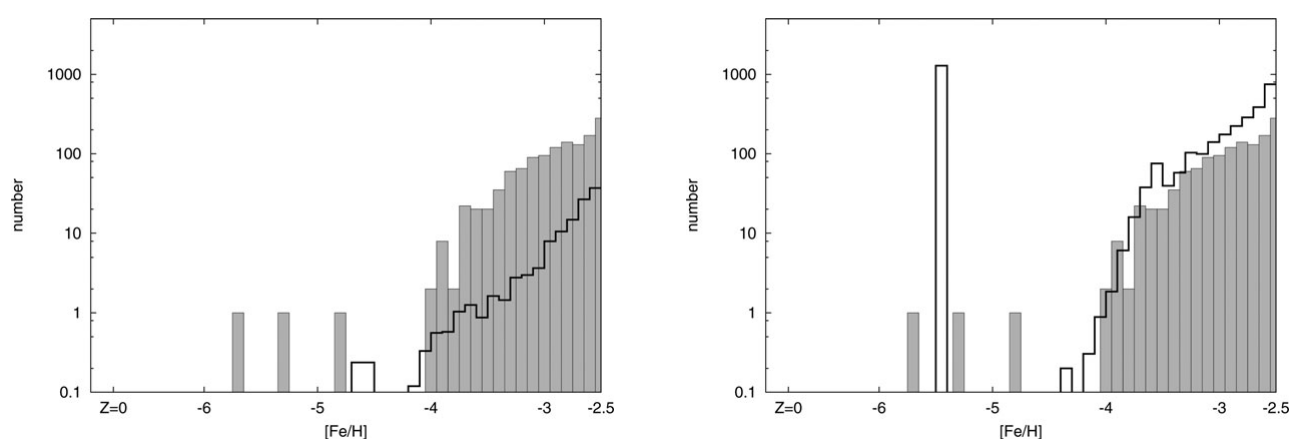

Figure 3. Effect of PISN. left: First stars in all mini-halos assumed to become PISN. right: First stars in mini-halos formed with $[\mathrm{Fe} / \mathrm{H}]<[\mathrm{Fe} / \mathrm{H}]_{c}-5.5$ become PISN.

metallicity of main-sequence Pop. III stars is distributed around $[\mathrm{Fe} / \mathrm{H}] \sim-3$ to -4 , and is diluted to $[\mathrm{Fe} / \mathrm{H}] \sim-5$ to -6 when they evolve to giants. This result implies that HMP stars are possibly polluted Pop. III stars that accrete iron group elements onto their surface from the ISM.

\subsection{Pre-enrichment by the first supernova}

We considered the effect of pair-instability supernovae (PISNe) for Pop. III stars that produce huge explosion energies sufficient to blow off the host mini-halo. We assume that $10 M_{\odot}$ of iron is ejected by PISNe and is mixed into the galactic matter instantaneously and homogeneously. Figure 3 shows the resultant MDF with the outflow of gas and metals by PISNe taken into account. At first, we assume that a single PISNe event occurs in all mini-halos (see left panel). In this case, overproduction of metals by PISNe reduces the number of EMP stars and the resultant MDF is inconsistent with observations.

We also assume the case that the PISNe of first stars occur in the mini-halo with $Z \leqslant Z_{c r}=10^{-5.5} Z_{\odot}$ and $T_{v i r}<10^{4} \mathrm{~K}$ (see right panel). In this model, the theoretical $\mathrm{MDF}$ with $[\mathrm{Fe} / \mathrm{H}]>-5$ is quite similar to Figure 1 and consistent with observations. However, too many stars with $Z \sim Z_{c r}$ are formed instead of stars with $Z=0$. In 
any case, if the PISNe contribute to the pre-enrichment of the Galaxy, the observed abundances of HMP stars should reflect the yields of PISNe, although the observations do not support this.

These results are inconsistent with observations but we cannot reject the possibility that some PISNe occur in the early universe because their yields may be masked by accreted ISM in the currently observed HMP stars. Our results will provide some constraints on the understanding of the first supernovae and pre-enrichment history of the Galaxy.

\section{Conclusions}

We modelled the formation history of EMP stars with realistic merging history and simple chemical evolution. Theoretical MDFs of EMP stars are calculated and compared with observations. Our hierarchical model naturally explains the metallicity cut-off at $[\mathrm{Fe} / \mathrm{H}] \sim-4$. On the other hand, the theoretical MDF with $[\mathrm{Fe} / \mathrm{H}] \lesssim-5$ is inconsistent with observations. This suggests that the formation process and mass distribution of stars with $[\mathrm{Fe} / \mathrm{H}] \lesssim-5$ may differ from more metal-rich populations.

Our estimate of chemical enrichment implies that HMP stars are formed before the first supernovae in their host halos. Considering the effect of surface pollution in the hierarchical model, we show that they are possibly polluted Pop. III stars. We also consider the effect of pre-enrichment by PISNe, but these models fail in reproducing the observed MDF.

\section{References}

Abel, T, Bryan, G. L., \& Norman, M. L. 2002, Science, 295, 93

Aoki, W., Beers, T. C., Christlieb, N., Norris, J. E., Ryan, S. G., \& Tsangarides, S.2007 ApJ, 655,492

Beers, T. C., Preston, G. W., \& Shectman, S. A. 1992, AJ, 103, 1987

Beers, T. C., Christlieb, N., Norris, J. E., Bessell, M. S., Wilhelm, R., Allende P. A., Yanny, B., Rockosi, C., Newberg, H. J., Rossi, S., \& Lee, Y. S. 2005, Proc. IAUS 228, 175

Bond, J. R., Cole, S., Efstathiou, G., \& Kaiser, N. 1991, ApJ, 379, 440

Christlieb, N., Green, P. J., Wisotzki, L., \& Reimers, D. 2001, A\& 4 , 375, 366

Christlieb, N., Bessell, M. S., Beers, T. C., Gustafsson, B., Korn, A., Barklem, P. S., Karlsson, T., Mizuno-Wiedner, M., Rossi, S. 2002, Nature, 419, 904-906

Frebel, A., Aoki, W., Christlieb, N., et al. 2005, Nature 434, 871-873

Fujimoto, M. Y., Sugiyama, K., Iben, I. Jr., \& Hollowell, D. 1995, ApJ , 444, 175

Fujimoto, M. Y., Ikeda, Y., \& Iben, I. Jr. 2000, ApJ, 529, L25

Karlsson 2006, $A \mathscr{E} A$,

Karlsson 2006, ApJl, 641, L41

Komiya, Y., Suda, T., Minaguchi, H., Shigeyama, T., Aoki, W., \& Fujimoto, Y. M. 2006 ApJ 658,367

Komiya, Y., Suda, T., \& Fujimoto, Y. M. 2008 ApJ submitted.

Komiya, Y., Suda, T., Asao, H., \& Fujimoto, Y. M. 2008, in preparation.

Lacey, C., \& Cole, S. 1993, MNRAS, 262, 627

Nishi, R., \& Susa, H. 1999, ApJ, 523, L103

Norris, John E., Christlieb, N., Korn, A. J., Eriksson, K., Bessell, M. S., Beers, Timothy C., Wisotzki, L., Reimers, D. 2007 ApJ, 670, 774

Salvadori, S., Schneider, R., \& Ferrara, A. 2006, MNRAS, 381, 647

Schaerer, D 2002, A\&A A, 382, 28

Somerville, R. S., \& Kolatt, T. S. 1999, MNRAS, 305, 1

Spite, M. et al. 2005, A\& A , 430, 655 
Suda, T., Aikawa, M., Machida, M. N., Fujimoto, M. Y., \& Iben, I. Jr. 2004, ApJ, 611, 476 Suda, T. et al. 2008, PASJ, in press

Tegmark, M., Silk, J., Rees, M. J., Blanchard, A., Abel, T., \& Palla, F. 1997, ApJ, 474, 1 Tumlinson, J. 2006, ApJ, 641, 1

Tominaga, N., Umeda, H., \& Nomoto, K. 2007, ApJ, 660, 516

Umeda, H. \& Nomoto, K. 2003, Nature, 422, 871 